\title{
Cosmic Explosions (Optical)
}

\author{
S. R Kulkarni \\ Caltech Optical Observatories, Pasadena, CA 91125, USA \\ email: prkulkarni@mac.com
}

One of the principal motivations of wide-field and synoptic surveys is the search for, and study of, transients. By transients I mean those sources that arise from the background, are detectable for some time, and then fade away to oblivion. Transients in distant galaxies need to be sufficiently bright as to be detectable, and in almost all cases those transients are catastrophic events, marking the deaths of stars. Exemplars include supernovæ and gamma-ray bursts. In our own Galaxy, the transients are strongly variable stars, and in almost all cases are at best cataclysmic rather than catastrophic. Exemplars include flares from $\mathrm{M}$ dwarfs, novæ of all sorts (dwarf novæ, recurrent novæ, classical novæ, X-ray novæ) and instabilities in the surface layers of stars such as $\mathrm{S}$ Dor or $\eta \mathrm{Ca}$ rina. In the nearby Universe (say out to the Virgo cluster) we have sufficient sensitivity to see novæ. In $\S 1$ I review the history of transients (which is intimately related to the advent of wide-field telescopic imaging). In $\S 2$ I summarize wide-field imaging projects, and I then review the motivations that led to the design of the Palomar Transient Factory $(\mathrm{PTF}) \dagger$. Next comes a summary of the astronomical returns from PTF $(\S 3)$, and that is followed by lessons that I have learnt from PTF $(\S 4)$. I conclude that, during this decade, the study of optical transients will continue to flourish (and may even accelerate as surveys at other wavelengths - notably radio, UV and X-ray - come on-line). Furthermore, it is highly likely that there will be a proliferation of highly-specialized searches for transients. Those searches may well remain active even in the era of LSST ( 55$)$. I end the article by discussing the importance of follow-up telescopes for transient object studies - a topical issue, given the Portfolio Review that is being undertaken in the US.

\section{History: Phase Space}

One could say that large surveys of the sky for star positions, stellar photometry and stellar classification (via low-resolution spectroscopy) effectively define the start of the modern era of astronomy. The early returns resulting from the discovery and study of variable stars were stunning. RR Lyrae and Cepheid variables proved to be rather precise yardsticks, and astronomers came to appreciate the physical scale of our Galaxy and eventually the physical scale of the Local Universe.

All transients were initially classified as "nova stella" or new stars, and the abbreviation "novæ" came to be applied specifically to just classical novæ. One of the main goals of the study of novæ was to determine a distance to the Andromeda galaxy using the fact that fast novæ are, on average, brighter than the slow ones. Thus luminosity is converted to time-scale (which, for modest velocities, can be regarded as independent of frame). A large amount of Mt. Wilson 60-inch telescope time was spent by E. Hubble studying novæ in M31.

$\dagger$ PTF is a collaboration of the following entities: California Institute of Technology, Lawrence Berkeley National Laboratory (LBNL), Weizmann Institute of Sciences, Las Cumbres Observatory Global Telescope, Columbia University, Oxford University, Infrared Processing \& Analysis Center (IPAC) and UC Berkeley. LBNL is responsible for the image subtraction pipeline, IPAC for the photometric pipeline and U. C. Berkeley for the classification engine. 


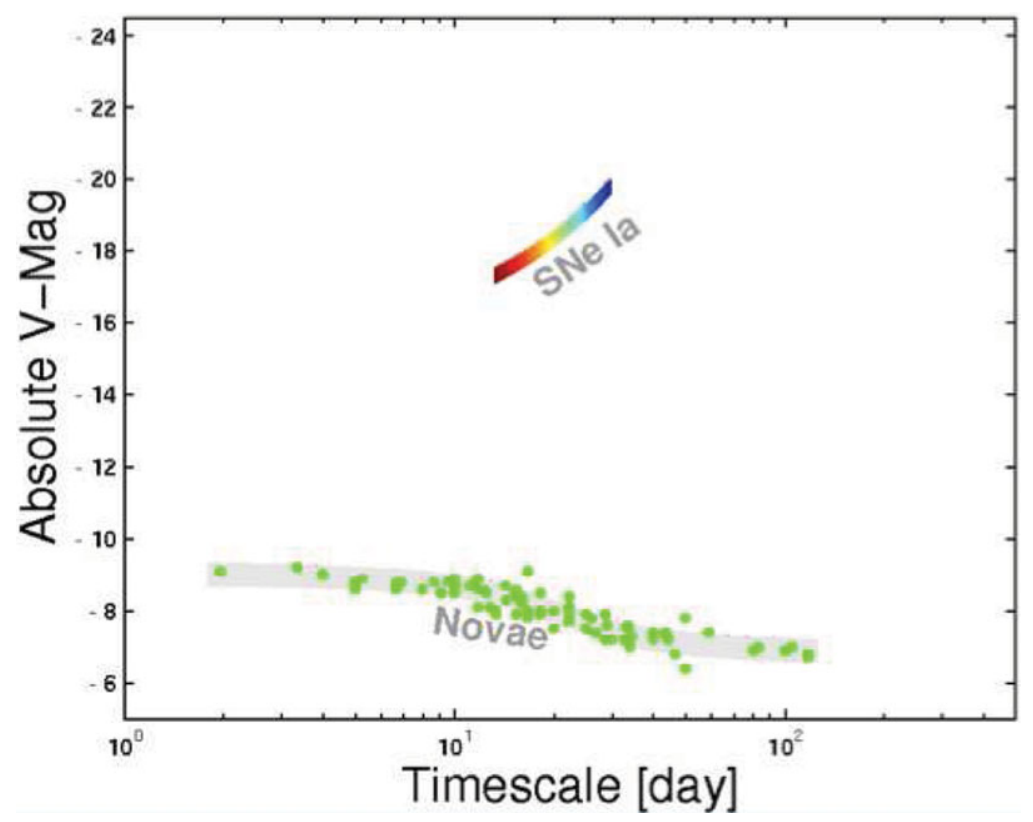

Figure 1. Phase space of classically known non-relativistic explosions in the Zwicky era: novæ and Type Ia supernovæ. The horizontal axis is the decay time-scale and the $y$-axis is the peak luminosity but shown as absolute magnitude in the $V$-band. Type II explosions have not been included because the explosion physics is mediated and hence masked by the envelope. Notice the wide gap between novæ and supernovæ.

The modern era of transients, with a focus on controlled cadence and a physicsbased enquiry, began with F. Zwicky and W. Baade. Recognizing the importance of the then newly-invented "Schmidt" type wide-field telescope $\nmid$ Zwicky obtained funds from a wealthy family in Pasadena and had constructed an 18-inch telescope using the Schmidt-camera design. The "P18" was the first telescope on Palomar mountain $\ddagger$. The first major result was the recognition of two distinct families: classical novæ and "supernovæ" (Baade \& Zwicky 1934); see Fig. 1. In their very next paper the authors made the bold conjecture that supernovæ mark the transmutation of an aging star into a neutron star, a very compact object (itself a novel hypothesis first proposed by L. Landau in 1931). The resulting enormous release of gravitational binding energy would accelerate some particles to relativistic velocities or cosmic rays. Next, thanks to the systematic survey carried out by Zwicky, families of supernovæ were recognized (Type I and Type II have certainly survived the passage of time) $\uparrow$.

The success of P18 motivated Zwicky to seek a larger Schmidt-type telescope, and that led to the 48-inch telescope ("P48") $\dagger$. It saw first light at about the same time as the Palomar 200-inch (circa 1948). P48 undertook the ambitious and comprehensive Northern hemisphere sky surveys (POSS-I and POSS-2). The photographic plates were

$\dagger$ Alerted to Zwicky by W. Baade, who knew the inventor Bernhard Schmidt.

$\ddagger$ The telescope still exists and will soon be moved to the Palomar Museum; the P18 dome is now our aeronomy centre, housing a polar telescope for seeing monitoring.

I It would be interesting to revisit types III, IV and V that Zwicky had proposed.

$\dagger$ Currently known as the Samuel Oschin telescope after a benefactor whose gift allowed the telescope to be rejuvenated. 
replaced by CCDs with ever increasing sophistication: "3-banger", QUEST (Baltay et al. 2007), CFH12K (Cuillandre et al. 2001).

\section{The Era of (Optical) Synoptic Surveys}

It is clear that we are now well into the era of synoptic and/or wide-field astronomy. In the 1-m to 2-m category we have the Catalina Sky Survey, Palomar Transient Factory (PTF), Pan-STARRS-1, SkyMapper and La Silla-QUEST. In the 2-m to 4-m category we have the magnificent Canada-France-Hawaii MegaCam, the VLT Survey Telescope (VST), the One Degree Imager on WIYN (under construction) and Dark Energy Camera on the Blanco 4-m telescope (and expected to be commissioned during 2012). Finally, in the behemoth category we have Suprime-Cam (and soon Hyper Suprime-Cam, HSC) on the Subaru 8.3-m telescope (also expected to be commissioned during 2012). The ultimate in this category will be the Large Synoptic Survey Telescope, an 8.3-m telescope but equipped with an imager with a field of view about five times larger than that of HSC. All in all we have an impressive list of facilities and projects. Given the large to gigantic formats of the imagers (0.1-1 gigapixel) some of these projects have the capacity to keep the entire global astronomical community busy for this entire decade simply with undertaking follow-up or follow-along observations.

\section{The Palomar Transient Factory}

The Palomar Transient Factory (PTF) was motivated by two considerations (Rau et al. 2009). First was the exploration of transients in the sky. A phase-space diagram is useful to summarize the known phenomenology graphically. An explosion has several basic parameters: the energy of the explosion, the mass of ejecta, the velocity of ejecta, the rise time of the explosion, the peak luminosity, and the decay time of the explosion. Peak luminosity and decay time-scales are easily measured, and as such are favourable for the principal axes of the phase space of transients. Fig. 1 shows the "classical" phase space of explosive transients. We were keen to find objects in the nova-supernova "gap".

A second motivation was the great promise of entirely new areas of astronomy:

(a) high-energy cosmic rays,

(b) high-energy neutrinos,

(c) highest-energy photons, and

(d) gravitational wave astronomy.

What is common to all these fields (apart from the fact that they represent new frontiers for astronomy and are quite expensive) is that the sources of interest to these facilities are associated with truly spectacular explosions. Furthermore, the horizon (radius of detectability), for reasons either of optical depth (GZK cutoff; $\gamma \gamma \rightarrow \mathrm{e}^{ \pm}$) or of sensitivity, is limited typically to $100 \mathrm{Mpc}$. Moreover, these facilities provide relatively poor localization. Thus, for the fields in question the study of explosions in the Local Universe $(d \lesssim 200 \mathrm{Mpc})$ is critical for two reasons: for improving the localization via lowenergy observations (which usually means in the optical), and for identifying and filtering out the torrent of false positives (because the expected rates of sources of interest is a tiny fraction of the known transients).

In Fig. 2 we display the phase space informed by theoretical considerations and speculations. Basing our surmises on the history of our subject we should not be surprised to find, say a decade from now, that we were not sufficiently imaginative.

PTF (see Law et al. 2009) consists of two dedicated telescopes: the P48, with a large field of view $\left(7.2 \mathrm{deg}^{2}\right)$, acting as the Discovery Engine, and the Palomar 60-inch (P60) acting as the Photometric Engine. The former is equipped with the re-engineered 


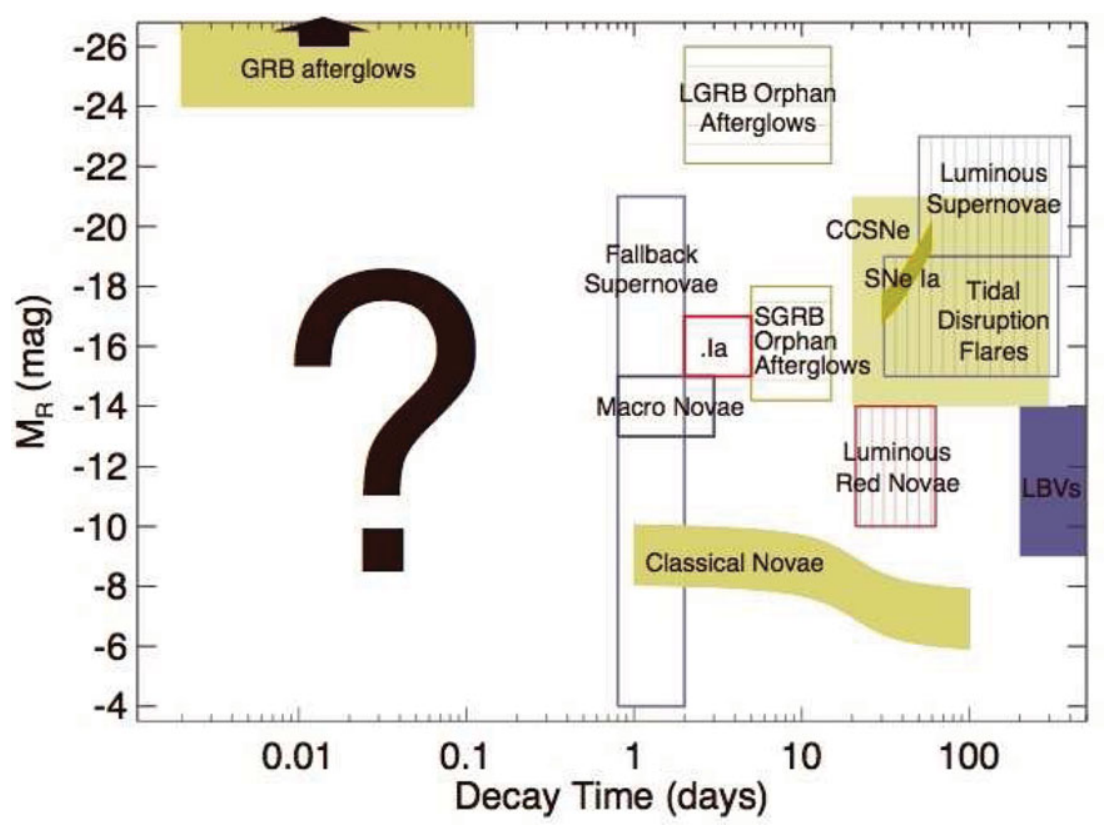

Figure 2. Theoretical and physically plausible possibilities are marked in the explosive transient phase space. The original figure is from Rau et al. (2009). The updated figure (to show the unexplored sub-day phase space) is from the LSST Science Book (v2.0).

CFH12K 96 Megapixel CCD mosaic, and the latter with a single 4 Megapixel CCD. The clarity afforded by the singular focus allowed us to optimize PTF for transient studies. Specifically, unlike some projects such as SDSS SN search, PS-1 and SkyMapper, we undertake the search for transients in a single band ( $R$ band during most of the month and $g$ band during the darkest period). This simplification alone gives us a factor of five more target throughput.

Next, we adopted a "No Transient Left Behind" strategy. Specifically, a transient without any additional information (such as low-resolution spectroscopy) does not in most instances represent a meaningful advance. After all, it is relatively easy to discover ageing supernovæ, the occasional flare from the numerous dwarf stars, a burp from an accreting system and so on. To address this important issue we employ the P60 to obtain 3 -colour photometry to classify crudely the transient and use a variety of criteria for relatively prompt low-resolution spectroscopy on an armada of larger telescopes (the Palomar 200-inch, the KPNO 4-m, the WHT 4.2-m and the Lick 3-m). As a result we have amassed a set of nearly 1500 spectroscopically classified supernovæ of which a good fraction was detected prior to maximum.

Given that follow-up is at premium, having a small sample of transients with desired properties is more valuable than a large sample of transients with a pot-pourri of properties. Thus, the choice of pointings and cadence control are critical. We have scoured around the sky for regions with large local over-densities $(d \lesssim 200 \mathrm{Mpc})$. The special hundred PTF pointings contain 4 times more light than randomly chosen pointings (Kasliwal 2011). Cadence control is even more important. For instance, the logistics of obtaining UV spectra of Type Ia supernovæ require that the supernovæ be identified 10 to 14 days prior to peak. In 2010 we focused on finding such supernovæ, and we now have three dozen HST UV spectra of nearby Type Ia's - an order of magnitude increase in 
the sample size. Our sample enables us to study rest-frame spectra of both nearby and cosmological Type Ia supernovæ (Cooke et al. 2011).

Cadence control is essential for decreasing the latency between detection (by which I mean that a transient has been identified with a reliable degree of certainty) and discovery (by which I mean that the astronomer has a useful idea of the nature of the transient). At the very minimum we should know if the source is Galactic or extra-galactic. At the next level, it would be useful to have the first estimate of sub-typing (e.g., flare star/DN/CV; Type Ia/Ibc/II SN)). During 2011 we made efforts to decrease the latency and were (very luckily) rewarded with the discovery of a Type Ia supernova in the very local Universe (PTF11kly in Messier 101) just 10 hours after the explosion (Nugent et al. 2011). The proximity and near natal discovery allowed us and other astronomers to shed valuable new light on the progenitor of a Type Ia supernova.

With 38 refereed publications to date† (a mean rate of 1 publication per month) PTF has been productive. PTF supported the thesis projects of three students. In addition to the two results summarized above, key discoveries and findings include the clear identification of a class of luminous supernovæ whose spectra show no hydrogen (Quimby et al. 2011), the gradual "colouring of the phase space", as shown well in Fig. 1 of Kasliwal (p. 62), and an apparent new type of nuclear event (PTF10iya; Cenko et al. 2011).

\section{Lessons Learnt}

- First and foremost, a clear vision for the project must be articulated. The statement that it behooves astronomers to explore phase space (wrongly attributed to Zwicky) is frequently heard at august meetings as the one we have just had. Even a careless reading of Zwicky's morphological approach to problems stresses the importance of a sensible exploration of phase space and consistency with physics. In this regard, Fig. 1 on page 62 was an essential exercise in motivating PTF.

- Merely identifying a transient does not constitute a discovery. We are now operating in a régime in which transients are a dime a dozen. Going forward, culling either by brute force (spectroscopy) or by cadence control is essential.

- A horizontal structure is best suited to the pursuit of transients. Such a structure allows for key project teams that are in effect independently operated to undertake followup efficiently and rapidly. However, nature is rarely so neatly organized. Undoubtedly, there will be overlap by the way of objects and interests. A strong leadership is essential to resolve such situations.

- Software pipelines have to be fully functional before the searches begin. This statement is a truism, but apparently is not always satisfied in practice.

- The collaboration should be flexible enough to change search strategies in order to respond to new transients discovered at the edge of a currently studied phase space. In 2009 and 2010 we focused on a standard SN programme, planning for a five-day cadence. On crossing the 1,000-SN mark we increased the cadence; with each ratcheting down of the latency we had interesting finds, and the final prize was PTF11kly in M101 (Nugent et al. 2011).

\section{Future: Beetles and Behemoths}

The following question inevitably arises at meetings such as this: is there a need for more synoptic surveys since even a modest-aperture synoptic survey can generate more

$\dagger$ http://www.astro.caltech.edu/ptf 
data than can be followed up? This is a meritorious question and my answer is that there is considerable room for highly-focused projects. I list three examples. (1) The Catalina Sky Survey, a survey based on 1-m telescope equipped with a routine CCD detector, is remarkable for its harvest of NEOs and pinpointing (with considerable headsup) the fiery entry of 2008TC3 (Boattini et al. 2009). (2) PTF, with its laser-like focus on transients has, as remarked in $\S 3$, been very productive and has also made noticeable contributions to our field. Finally, (3) the CFHT SN Legacy Survey (CFHT; Conley et al. 2011) has made deep contributions to Ia cosmology and the SDSS Stripe 82 project has made contributions across board-SN cosmology, AGN variability, stellar variability, tidal disruption events (e.g., van Velzen et al. 2011). Looking at these gains I would venture to say that focused projects have the ability to trump much larger facilities which are subject to competing cadence demands.

The second reason why I remain bullish on the continued value of the current portfolio of synoptic surveys is that the systematic and sensible exploration and study of the phase space has only just begun. The phase space with decay time of less than a day (for which there are several plausible models) and the entire physics of the rise time of explosive transients is in effect wide open.

Finally, the transient game is currently dominated by optical synoptic facilities and projects. The next to join this club would be radio facilities at both metre and decimetre wavelengths (EVLA, LOFAR, MWA, upgraded GMRT, APERTIF, MeerKAT). With some luck, synoptic space-based projects may also happen during this decade (e.g., LIM$\mathrm{SAT} \dagger$ in the UV, reuse of WISE for thermal IR searches and a Lobster-type mission in the X-rays). One can easily imagine joint studies with optical facilities.

Projects such as PTF, CSS and PS-1 demonstrate that there is ample scope for 1-m to 2 -m-class surveys to continue well into this decade. The magnitude limit of 21 is ideally suited to classification spectroscopy (from which I do not see a simple escape). Going fainter is only an advantage if one has the ability to discriminate between the "unknown unknowns" against the dense fog of known transients. Recall that follow up at the 22-mag requires 6 times as much as follow up time (on the same telescope) as a 21-mag event.

However, as noted several times, detecting a transient is merely the first step. Progress only happens when follow-up observations can be undertaken. Thus, follow-up telescopes are an essential requirement for transient studies. Currently, in the US there is a debate over the future of existing facilities. It is easy (trivial) to make an argument for ever larger telescopes. However, for many fields consolidation of resources (with strong focusing of the same) is more important. Indeed, some of the great discoveries over the past two decades have come from concerted efforts on modest-sized telescopes (e.g., planets around normal stars). PTF is an example of repurposing existing telescopes for singular goals at a modest cost. $\dagger$ Both discovery (usually arising from focused or smaller facilities) and depth of understanding (usually requiring larger facilities) are essential. However, the balance has tipped far too much for larger telescopes.

So enthused am I with the prospects and promises of focused programmes (along with appropriate follow-up) that, along with my colleagues, I am now proposing a secondgeneration of PTF. We propose to equip fully the entire focal plane of P48 (40 $\left.\mathrm{deg}^{2}\right)$, build an efficient IFU ultra-low resolution $(R=\lambda / \Delta \lambda \sim 100)$ spectrograph $\ddagger$ optimized for classification, and a Rayleigh-scattering AO system $\Phi$ for sensitive photometry even in

$\dagger$ This is a cluster of UV telescopes with a total of 1,000 square degrees and promoted by an ad hoc group of astronomers from Israel, Caltech, India and Canada.

$\dagger$ The capital and 4-year operating cost of PTF is under $\$ 3 \mathrm{M}$.

$\ddagger$ http://sites.google.com/site/nickkonidaris/sed-machine

I http://www.astro.caltech.edu/Robo-AO 
crowded host galaxy fields. In addition to P60 we are seeking another telescope so that the P48 along with these two telescopes will be one of the first new-generation transient facilities. The primary focus of this facility will be to probe the sub-day phase space (marked by a big "?" in Fig. 2). I propose to name it the "Zwicky Transient Facility" (ZTF) after the founder of our field. With some effort it should be possible for this facility to see first light by 2015. Should interesting sub-minute bursts be discovered, then one can imagine upgrading ZTF with CMOS. It is thus hoped that ZTF, a mere beetle, will prove productive and interesting even as general-purpose behemoths come on line.

\section{Acknowledgements}

I would like to acknowledge the National Astronomy Observatory of Japan and the Japan Society for Promotion of Science for hosting my sabbatical stay in Japan during which period this article was completed.

I would like to thank all the members of the PTF experiment. It is their hard work and creativity that has made PTF productive.

I would further like to acknowledge the key role played by the following in the PTF: L. Bildsten, J. Bloom, S. B. Cenko, A. Gal-Yam, M. Kasliwal, R. Laher, N. Law, P. Nugent, E. Ofek, R. Quimby and J. Surace. The hard-working and knowledgeable staff of Caltech Optical Observatories made it possible for rapid re-engineering of the focal plane of P48 and refurbishment of P48. The very low down-time, despite the age of P48 and P60, speaks superbly of the deep knowledge and hard work of the Palomar Mountain staff. D. Fox, B. Cenko and M. Kasliwal were in charge of the automation, robotization and optimization of P60, and E. Ofek was (and continues to be) the sequencer for P48.

PTF got a great start with CFH12K, and I sincerely appreciate the generosity of the builders of CFH12K, Christian Veillet (the then Director of CFHT) and the CFHT Corporation. I would like to thank D. Frail, G. Helou and T. Prince for helping me debate strategies in this rapidly evolving field. Finally, I would like to acknowledge and thank W. Rosing, whose initial "investment" in PTF allowed me to garner funds from other parties and complete the funding for PTF in record time.

\section{References}

Baade, W., \& Zwicky, F. 1934, PNAS, 20, 254

Baltay, C., et al. 2007, PASP, 119, 1278

Boattini, A., et al. 2009, AAS-DPS, 41, \#9.02

Cenko, S. B., et al. 2011, arXiv1103.0779

Cooke, J., et al. 2011, ApJ, 727, L35

Conley, A., et al. 2011, ApJS, 192, 1

Cuillandre, J.-C., Starr, B., Isani, S., \& Luppino, G. 2001, ExA, 11, 223

Kasliwal, M. M. 2011, PhD Thesis, Caltech

Law, N. M., et al. 2009, PASP, 121, 1395

Nugent, P. E., et al. 2011, Nature, 480, 344

Quimby, R. M. et al. 2011, Nature, 474, 487

Rau, A., et al. 2009, PASP, 121, 1334

van Velzen, S., et al. 2011, ApJ, 741, 73 